\title{
Cricket pace bowling: The trade-off between optimising knee angle for performance advantages $v$. injury prevention
}

\author{
B Olivier, ${ }^{1} \mathrm{PhD}$; A V Stewart, ${ }^{1} \mathrm{PhD}$; A C Green, ${ }^{2} \mathrm{MSc}(\mathrm{Physiol}) ;$ W McKinon, ${ }^{2} \mathrm{PhD}$ \\ ${ }^{1}$ Department of Physiotherapy, School of Therapeutic Sciences, Faculty of Health Sciences, University of the Witwatersrand, Johannesburg, South Africa \\ ${ }^{2}$ School of Physiology, Faculty of Health Sciences, University of the Witwatersrand, Johannesburg, South Africa
}

Corresponding author: B Olivier (Benita.Olivier@wits.ac.za)

\begin{abstract}
Background. The cricket pace bowler utilises various strategies, including a more extended front knee angle, to achieve optimal performance benefits. At times this is done to the detriment of injury prevention.

Objective. To investigate the relationship between three-dimensional (3D) knee kinematics during pace bowling action, injury incidence and bowling performance at the start and end of a cricket season.

Methods. Knee angle and ball release (BR) speed of injury-free premier league (club level) cricket pace bowlers over the age of 18 years were measured at the start and end of the cricket season. Kinematic, injury- and bowling performance-related (BR speed and accuracy) data were analysed using paired and independent Student's $t$-tests, Pearson's correlation coefficient, $\chi^{2}$ test and a two-way analysis of covariance with repeated measures.

Results. Thirty-one bowlers participated in this study, and kinematic data of a subset of 17 were analysed. Nine bowlers (53\%) sustained injuries during the cricket season. No statistically significant relationship was found between knee angle and injury. Bowlers who did not sustain an injury bowled with more knee flexion at the start of the season (mean (standard deviation) $157.07^{\circ}\left(12.02^{\circ}\right)$ ) than at the end of it $\left(163.95^{\circ}\left(6.97^{\circ}\right)\right)(p=0.01)$. There was no interaction between accuracy and knee angle. There was a good to excellent inverse correlation between BR speed and knee angle among bowlers who remained injury free $(r=-0.79 ; p=0.18)$.

Conclusion. Bowlers who remain injury free during the course of the season may use strategies other than the front knee angle to facilitate high BR speeds. Technique-related variables which are more 'protective' against injuries while allowing for higher BR speeds should be further investigated among bowlers.
\end{abstract}

S Afr J Sports Med 2015;27(3):76-81. DOI:10.7196/SAJSM.8111

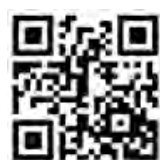

Ball release (BR) speed and bowling accuracy are two variables that contribute to the performance of a cricket pace bowler. A batsman has only a few milliseconds to judge the flight of the ball if a high BR speed is successfully achieved. In addition to high BR speeds, if a pace bowler can predict the pitch of the ball, such accuracy affords him a useful tool which allows him to strategically plan dismissal of a batsman. ${ }^{[1]}$ The strain applied to a bowler's body to facilitate greater BR speeds and accuracy are known to predispose the bowler to injury. ${ }^{[2,3]}$ Therefore the optimisation of bowling performance and prevention of injury are necessary among cricket pace bowlers.

The relationship between technique-related three-dimensional (3D) kinematics of the pace bowling action and BR speed has been widely investigated in once-off cross-sectional studies. Front knee angle during the delivery stride of the pace bowling action has been associated with higher BR speeds ${ }^{[4,5]}$ In addition, a more extended knee contributes to higher BR speed by increasing the radial distance between front foot contact and the extended bowling arm, which results in a greater tangential endpoint velocity. ${ }^{[6]}$ However, there is still controversy around the relationship between BR height and BR speed. $^{[5]}$

Studies investigating the association between bowling accuracy and technique-related variables are scarce. ${ }^{[7,8]}$ In addition to studies investigating BR speed, a few have investigated the association between front knee angle and injury, and found that a more extended knee during the power phase of the pace bowling action, when ground reaction forces are exceptionally high, is associated with injury. ${ }^{[9,4,2]}$ The interrelationship between proximal (lower back) and distal body segments (knee) has been confirmed by Crewe et al. ${ }^{[3]}$ who found an association between a more extended front knee during the front foot contact phase and lumbo-pelvic shear forces.

Since lower quarter injuries, lower back and lower limb injuries are extremely common in pace bowlers, ${ }^{[10-12]}$ the investigation of lower quarter injuries may provide useful insights. The comparison of kinematics at the start and at the end of the season, and between injured and non-injured players may give valuable information on the cause and effect of knee kinematics during bowling action.

The rarity of longitudinal studies assessing technique-related variables and their possible contribution to speed and accuracy prompted this study. Therefore the aim of this study was to investigate the relationship between 3D knee kinematics during the pace bowling action, injury incidence and bowling performance at the start as well as at the end of a cricket season.

\section{Methods}

Study design, setting and participants

This is a descriptive study with a longitudinal component. Data collection took place at the University of the Witwatersrand's indoor 
cricket nets. Premier league cricket pace bowlers playing for cricket clubs in south Gauteng Province, South Africa, were randomly invited to participate in this study. Injury-free pace bowlers over the age of 18 years were included in this study. Bowlers who have undergone previous surgery to the spine or limbs were excluded from this study. A pace bowler was defined as a bowler bowling at a speed of $120 \mathrm{~km} / \mathrm{h}$ or more, ${ }^{[12]}$ and who averaged more than five overs bowled in matches played during any of the previous two seasons. ${ }^{[13]}$

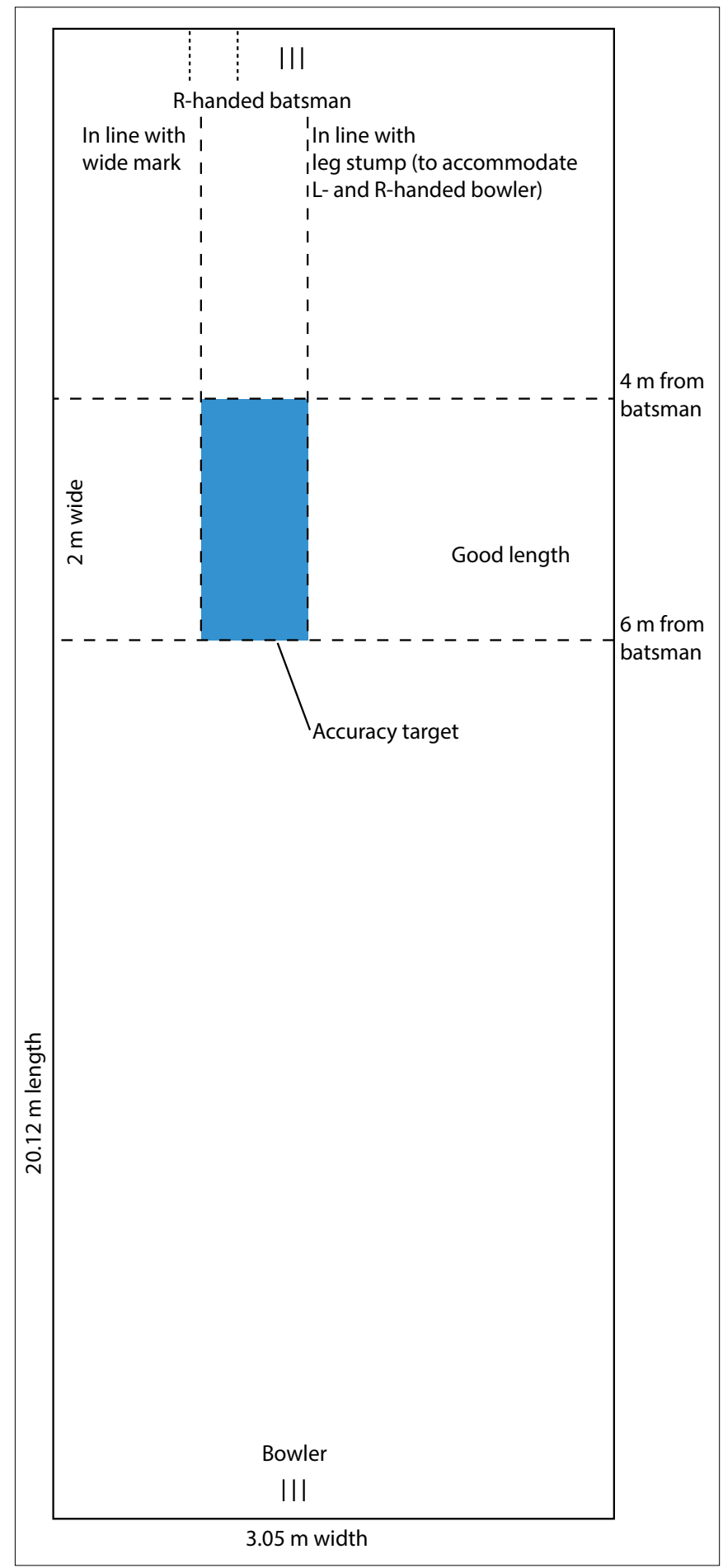

Fig. 1. Accuracy target on the pitch.

\section{Injury surveillance}

Injuries (status, nature and prevalence) were monitored monthly through use of a standardised (self-reporting) questionnaire ${ }^{[14]}$ throughout the duration of the cricket season. The self-report injury questionnaire enquired on the status, nature, mechanism and management of injuries. An injury was defined as a 'musculoskeletal condition that resulted in loss of at least one day of sporting activity or that occurred during a sporting activity that required medical attention or which forced the bowler to quit the activity.[14]

\section{Bowling performance: BR speed and accuracy}

BR speed was captured at the start and end of the season using a handheld radar gun (Stalker ATS, USA) positioned $180^{\circ}$ behind the BR point ${ }^{[5]}$ Classification of bowlers according to speed was as follows: medium pace $(120-129 \mathrm{~km} / \mathrm{h})$; medium fast pace $(130-139 \mathrm{~km} / \mathrm{h})$ and fast pace $(\geq 140 \mathrm{~km} / \mathrm{h}) .{ }^{[12]}$

Bowling accuracy was assessed according to a categorical scale measuring the ability of the bowler to pitch the ball in two target blocks, namely a marked target on the pitch (Fig. 1) ${ }^{[1]}$ and a target behind the stumps (Fig. 2). ${ }^{[7]}$ If a bowler pitched the ball in none, one or both of the two target blocks, he obtained a score of 0,1 or 2 out of 2 respectively. ${ }^{[7,1]}$ Each bowler bowled with a new $156 \mathrm{~g}$ cricket ball (Kookaburra Sport Ltd, South Africa).

\section{Instrumentation and set-up}

Kinematic analysis considered three dimensions: length, breadth, and depth. Kinematic variables were assessed at the start and the end of an 8-month cricket season. Kinematic data were captured using five high-speed digital cameras (PixeLINK PL-A741, USA) which recorded at 85 frames per second. Cameras were positioned around a capture volume of $12.76 \mathrm{~m}$ long, $4.25 \mathrm{~m}$ wide and $2.08 \mathrm{~m}$ high. A mean

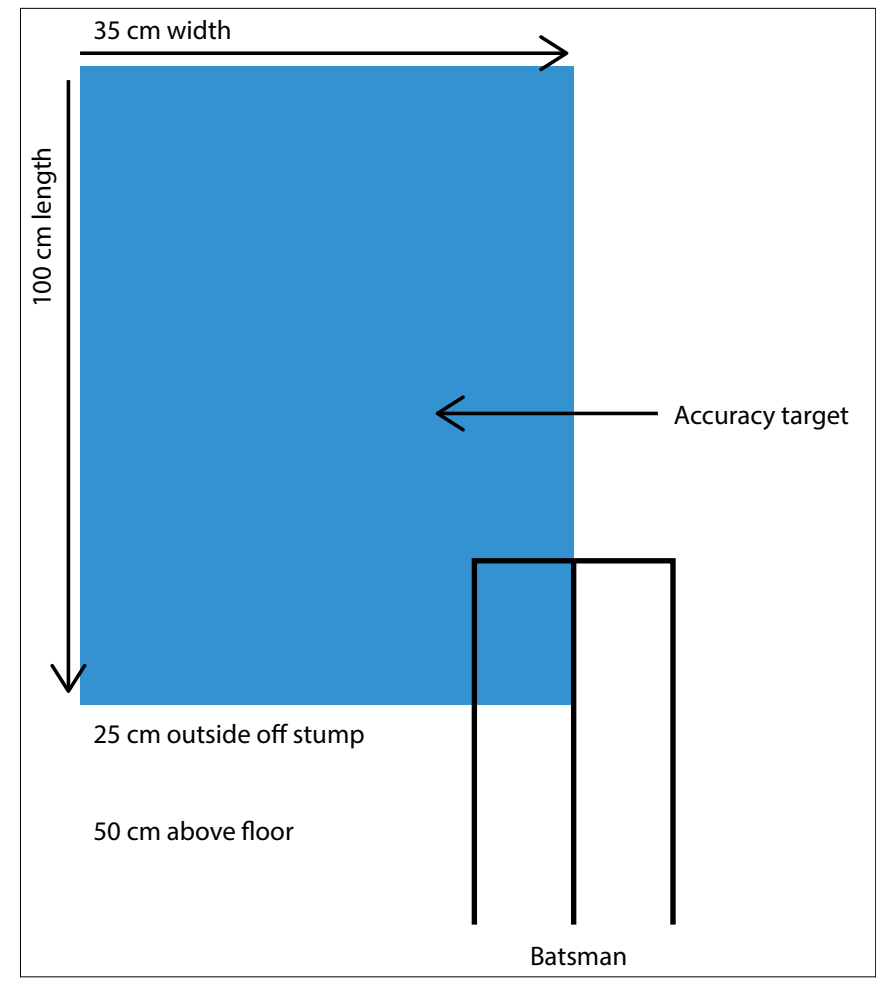

Fig. 2. Accuracy target behind the stumps. 
(standard deviation (SD)) residual error of marker position of less than $1.2(0.7) \mathrm{mm}$ was found.

\section{Procedures}

A pilot study was done with five bowlers whose data were not included in the main study due to minor changes to instructions to bowlers and order of data collection procedures. Bowlers first warmed up in their own accustomed manner, which included the opportunity to bowl six practice balls. Light-reflective markers were attached with double-sided adhesive tape to predetermined anatomical landmarks, as well as to the ball (Fig. 3). Each participant bowled six match-pace deliveries aimed at a right-hand batsman while being recorded. For each attempt BR speed and accuracy were measured.

\section{Ethical considerations}

Written informed consent was required and confidentiality was ensured. Ethical approval was obtained from the University of the Witwatersrand Human Research Ethics Committee.

\section{Data reduction and statistical analysis}

The power phase was defined from front foot placement (FFP) to BR. ${ }^{[2]} \mathrm{FFP}$ was identified as the first frame when the front (nondominant) toe marker reached its lowest position. BR was defined as the first frame observed where the ball was no longer in contact with the bowler's hand. ${ }^{[5]}$ All data for left-hand bowlers have been converted to read as data for right-hand bowlers for analytical standardisation. The delivery that obtained

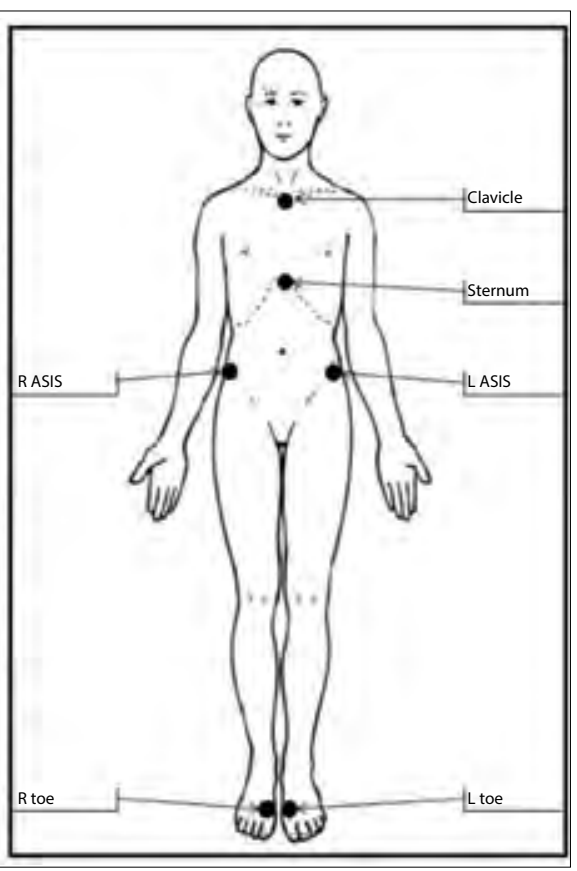

Fig. 3. Predetermined anatomical landmarks for marker placements. $(R=$ right; $L=$ left; $P S I S=$ posterior superior iliac spine; ASIS = anterior superior iliac spine.) the highest accuracy score in conjunction with the fastest BR speed was used for kinematic analysis of variables. Bowlers who sustained a lower quarter (lower back and lower limb) injury during the cricket season under review are referred to as 'injured', and those who remained injury free are referred to as 'non-injured'. Only non-contact injuries were included in the analysis. Data collected at the start of the season were referred to as preseason data and those collected at the end of the season as postseason data. Knee angles were classified into four different groups (basic classification), depending on the angle at FFP and BR (Table 1). Knee-angle classification categories were modified from those described by Bartlett et al. ${ }^{[6]}$ and Portus et al. ${ }^{[4]}$

Statistical analysis was conducted using SPSS version 22 (IBM, USA) using a twoway repeated measures analysis of covariance (ANCOVA) with an alpha level of 0.05 . There were two primary factors (FFP knee angle and BR knee angle), each with two levels (pre season and post season). The categorical variables, such as injury and accuracy, were calculated as between-subjects factors, while the other continuous variable (the average speed pre- and postseason) was calculated as a covariate. Additional analysis, namely paired-sample and independent $t$-tests when there were categorical variables and linear regression for purely continuous variables, were conducted to further investigate these interactions. Pearson's product moment correlation coefficient $(r)$ was used to identify relationships between continuous variables (kinematic variables and BR speed). Qualitative descriptions for the strength of the relationships were used to contextualise the relationships between continuous variables as follows: $r=0.00-0.25$ (little or no

Table 1. Knee angle $\left({ }^{\circ}\right)$ classification category ${ }^{\star}$

\begin{tabular}{|c|c|c|c|c|c|}
\hline \multicolumn{3}{|c|}{ Basic classification } & \multicolumn{3}{|c|}{ Further classification } \\
\hline & Knee angle at FFP & Knee angle at BR & & Knee angle at FFP & Knee angle at BR \\
\hline \multirow[t]{2}{*}{ Flexor } & $<170^{\circ}$ & $<170^{\circ}$ or $=\mathrm{FFP}$ & Flexor + & $<170^{\circ}\left(\right.$ e.g. $\left.158^{\circ}\right)$ & $<$ FFP $\left(\right.$ e.g. $\left.146^{\circ}\right)$ \\
\hline & & & Flexor - & $<170^{\circ}\left(\right.$ e.g. $\left.158^{\circ}\right)$ & $>$ FFP $\left(\right.$ e.g. $\left.168^{\circ}\right)$ \\
\hline Flexor-extender & $<170^{\circ}\left(\right.$ e.g. $\left.146^{\circ}\right)$ & $\geq 170^{\circ}\left(\right.$ e.g. $\left.174^{\circ}\right)$ & & & \\
\hline \multirow[t]{2}{*}{ Extender } & $\geq 170^{\circ}\left(\right.$ e.g. $\left.174^{\circ}\right)$ & $\geq 170^{\circ}$ or $=\mathrm{FFP}$ & Extender + & $\geq 170^{\circ}\left(\right.$ e.g. $\left.174^{\circ}\right)$ & $\begin{array}{l}>\text { FFP }\left(\text { e.g. } 178^{\circ}\right) \text {, or } \\
\text { into hyperextension }\end{array}$ \\
\hline & & & Extender - & $\geq 170^{\circ}$ (e.g. $\left.174^{\circ}\right)$ & $<\operatorname{FFP}\left(\right.$ e.g. $\left.170^{\circ}\right)$ \\
\hline Extender-flexor & $\geq 170^{\circ}\left(\right.$ e.g. $\left.174^{\circ}\right)$ & $<170^{\circ}\left(\right.$ e.g. $\left.146^{\circ}\right)$ & & & \\
\hline
\end{tabular}


Table 2. Knee angle $\left({ }^{\circ}\right)$ and BR speed $(\mathrm{m} / \mathrm{s})$ at the start and end of the cricket season, mean (SD)

\begin{tabular}{llllllll}
\hline & \multicolumn{3}{c}{ Pre season } & & \multicolumn{3}{c}{ Post season } \\
\cline { 2 - 4 } Variable & Non-injured $(\boldsymbol{n}=\mathbf{8})$ & Injured $(\boldsymbol{n}=\mathbf{9})$ & Total $(\boldsymbol{n}=\mathbf{1 7})$ & & Non-injured $(\boldsymbol{n}=\mathbf{8})$ & Injured $(\boldsymbol{n}=\mathbf{9})$ & Total $(\boldsymbol{n}=\mathbf{1 7})$ \\
\hline Knee angle at FFP $\left(^{\circ}\right)$ & $157(12)$ & $161(8)$ & $159(10)$ & & $164(7)$ & $160(11)$ & $161(9)$ \\
Knee angle at BR $\left(^{\circ}\right)$ & $143(21)$ & $140(21)$ & $141(20)$ & & $138(18)$ & $127(16)$ & $132(17)$ \\
BR speed $(\mathrm{m} / \mathrm{s})$ & $122(6)$ & $125(7)$ & $123(7)$ & & $122(8)$ & $116(24)$ & $119(18)$
\end{tabular}

relationship); $r=0.26$ - 0.50 (fair relationship); $r=0.51-0.75$ (moderate to good relationship); and $r>0.75$ (good to excellent relationship). ${ }^{[15]}$ A $\chi^{2}$ test was used to analyse the relationship between knee-angle classification category and accuracy (categorical data).

\section{Results}

\section{Participants and injury incidence}

Thirty-one fast, fast-medium and medium pace bowlers between the ages of 18 and 26 years (mean (SD) 21.8 (1.8) years) participated in the study. Twenty-six participants were right-handed and five were left-handed bowlers. Sixteen bowlers sustained one or more injuries during the course of the 8-month cricket season. All injured pace bowlers sustained at least one lower back and/or lower limb injury during the season as a direct result of the pace bowling action. Injuries comprised four lower back, four buttock and groin, two hamstring, four knee, one shin and four ankle injuries.

\section{Knee angle, injury incidence and bowling} performance

Owing to missing kinematic data, case-wise deletion of missing data was performed, and 14 bowlers were removed from the analysis. Analysis was performed on the remaining 17 bowlers, of whom eight were from the non-injured group and nine from the injured group.

Descriptive statistics for knee angle and BR speed are shown in Table 2. No statistically significant difference between preseason and postseason knee angle for the group as a whole was found for knee angle at FFP $(p=0.30)$ or at BR $(p=0.17)$. Average BR speed for the group was similar at the start and end of the season $(p=0.26)$. No statistically significant relationship was found between knee angle and injury (ANCOVA). Statistically significant within-subjects interactions were found between knee angle, BR speed and accuracy

Table 3. Statistically significant interactions between knee angle at FFP and BR bowling positions, BR speed and accuracy $(n=17)$

\begin{tabular}{|c|c|c|c|c|}
\hline Source of variance (within subjects) ${ }^{*}$ & df & SS & $F$ & $p$ \\
\hline Knee angle at FFP $\times$ knee angle at $\mathrm{BR} \times \mathrm{BR}$ speed & 1 & 775.436 & 7.068 & 0.029 \\
\hline $\begin{array}{l}\text { Knee angle at FFP } \times \text { knee angle at BR } \times \text { accuracy post } \\
\text { season }\end{array}$ & 2 & 1060.018 & 4.831 & 0.042 \\
\hline $\begin{array}{l}\text { Knee angle at FFP } \times \text { knee angle at } \mathrm{BR} \times \text { accuracy pre } \\
\text { season }\end{array}$ & 1 & 641.323 & 5.846 & 0.042 \\
\hline $\begin{array}{l}\text { Knee angle at FFP } \times \text { knee angle at BR } \times \text { accuracy pre } \\
\text { season } \times \text { accuracy post season }\end{array}$ & 1 & 634.264 & 5.781 & 0.043 \\
\hline
\end{tabular}

Error $8 \quad 1591.272$

$\mathrm{df}=$ degrees of freedom; $\mathrm{SS}=$ sum of squares

${ }^{*}$ The within-subjects effect and interaction of the repeated measure with independent variables; the between-subjects was computed for the independent measure, knee angle, and is not shown here owing to not being significant.

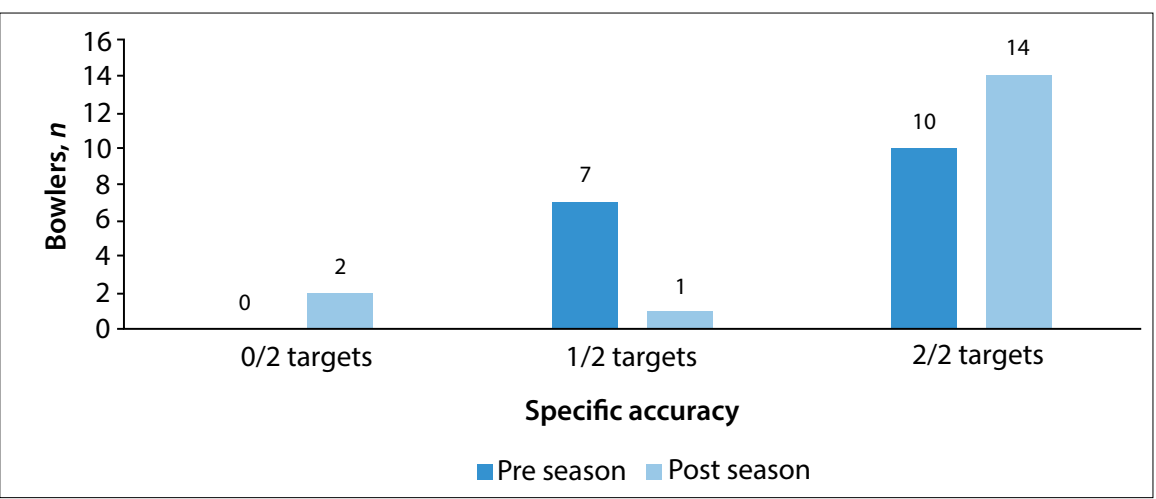

Fig. 4. Accuracy scores as measured at the start and end of the cricket season $(\mathrm{n}=17)$.

(ANCOVA) (Table 3). Paired $t$-tests revealed no statistically significant difference between knee angle as measured at the start of the season and knee angle at the end of the season in the injured group. However, a difference was found among bowlers who remained injury free during the course of the season, between the preseason knee angle of $157.07^{\circ}\left(12.02^{\circ}\right)$ and postseason knee angle of $163.95^{\circ}\left(6.97^{\circ}\right)$ in the FFP position of the bowling action $(p=0.01)$.

Most bowlers were successfully able to hit both of the accuracy targets (Fig. 4). The majority of bowlers were from the flexor classification category $(n=15)$, while one was classified as flexor-extender and one as extender. There was no interaction between accuracy and mean knee angle or between accuracy and knee-angle classification. There was a good to excellent inverse correlation between BR speed and knee angle in the noninjured group ( $r=-0.79 ; p=0.18$ ) (Fig. 5). No correlation was found between BR speed and knee angle for the injured group or the group as a whole.

\section{Discussion}

The objective of this study was to investigate the relationship between $3 \mathrm{D}$ knee kinematics, injury and bowling performance-related variables. The findings give useful insights into the role of a technique-related intrinsic factor in injury, BR speed and bowling accuracy. 


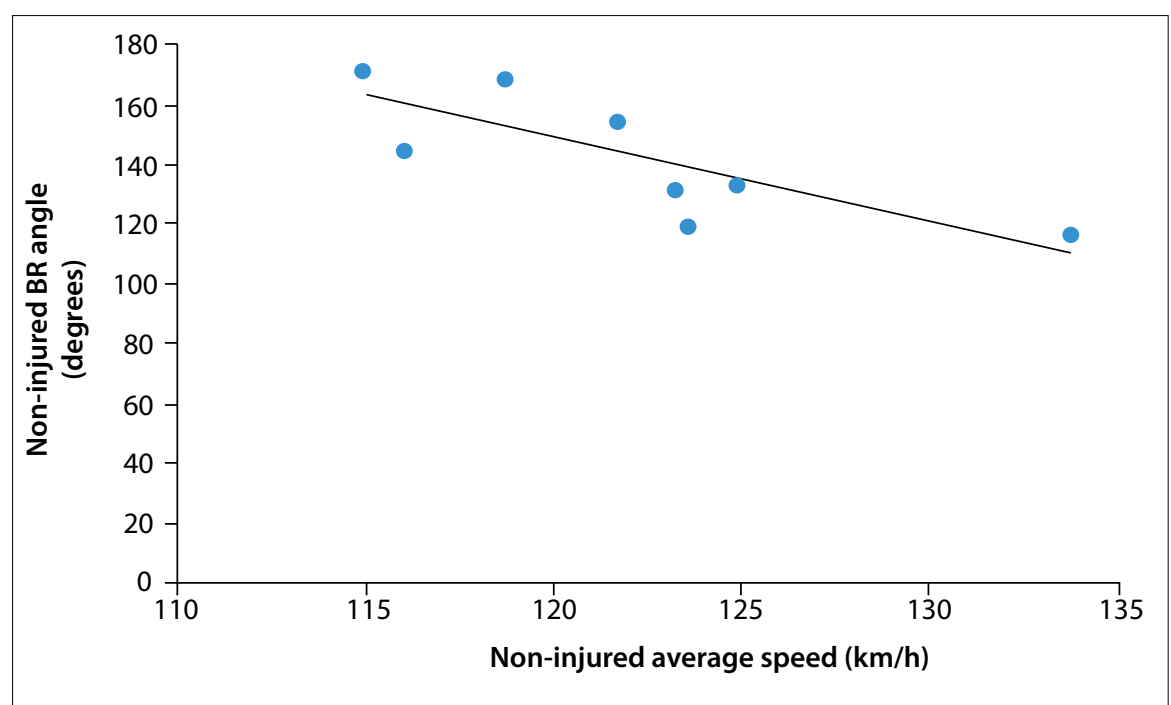

Fig. 5. Relationship between BR speed and knee angle in the non-injured at the start of the season $(\mathrm{n}=8)(\mathrm{r}=-0.79 ; \mathrm{p}=0.18)$.

The definition of injury used in this study is different to the current definition recommended for use in international injury surveillance. ${ }^{[13]}$ The recommended definition, which is currently under review, includes only match time-loss injuries, while the definition of injury used in this study was less stringent and allowed for less severe injuries to be identified. It is important to identify musculoskeletal injuries of all levels of severity because an injury which may not be severe enough to cause match time loss, may worsen if not identified early on. Also, only non-contact injuries sustained to the lower quarter - lower back and or lower limb - were included in the analysis of this paper which strengthens the findings, as it is specifically the non-contact injuries which can be influenced by technique modification. As the knee angle was studied, we preferred to focus only on lower back and lower limb injuries due to the distribution of force throughout the kinetic chain. ${ }^{[2,3]}$

In this study the ANCOVA did not identify injury status as statistically significant in the interaction with knee angle, which means there was no difference between mean knee angle in the injured v. the non-injured group. However, although no statistical difference was found in the injured group between knee angle measured at the start of the season compared with at the end of the season, the bowlers who did not sustain an injury during the season bowled with a more flexed knee at the start of the season compared with at the end of it. It may be that these bowlers who of the lower limb where the bowler may experience the injury-protective benefits of force attenuation due to the flexed limb, but not the apparent bowling-performance benefits associated with a straighter limb. ${ }^{[6,4]}$ However, in this study the opposite was true, where a correlation between a more flexed knee and higher BR speeds was found among non-injured bowlers (Fig. 5). No link between knee-classification categories and bowling performance (BR speed and accuracy) could be established due to the small numbers of bowlers in the flexor-extender and extender knee-classification categories.

No correlation was found between knee angle and BR speed for the group as a whole. Controversy exists in the literature with regard to the role of the front knee angle and BR speed. ${ }^{[7,4,5]}$ Wormgoor et al. ${ }^{[5]}(r=0.52$; $p=0.005)$ and Portus et al. ${ }^{[4]}$ (2004) $(r=0.37$; $p=0.02)$ found statistically significant, although moderate to low correlations between a front knee angle and BR speed, while Portus et al. ${ }^{[7]}$ (2000) did not find a statistically significant correlation. The sample sizes of those studies which indicated a statistically significant correlation were $28^{[5]}$ and $42^{[4]}$ bowlers respectively, while lower sample sizes were included in this study $(n=17)$ and that of Portus et al. ${ }^{[7]}(n=14)$. With larger samples even fair correlations will show statistical significance $(p<0.05)$. Also, previous studies did not investigate a potential correlation between BR speed and injury status separately, while in this study bowlers who remained injury free throughout the season bowled with a more flexed knee while still attaining higher BR speeds, as indicated by the good to excellent inverse correlation $(r=-0.79 ; p=0.18)$. Bowlers who remained injury free may have used strategies other than the front knee angle, like neuromusculoskeletal control such as balance and proprioception, to attain higher BR speeds. Both balance ${ }^{[14]}$ and proprioception ${ }^{[17]}$ have been associated with injury in previous research among pace bowlers. bowled with a more flexed knee at the start of the season.

In this study most bowlers formed part of the flexor category (Table 1), which means that they landed on a relatively flexed knee (in this case an angle of approximately $160^{\circ}$ ) and then flexed their knees a further $10^{\circ}$. Similar findings were observed by Wormgoor et al. ${ }^{[5]}$ Bartlett et al. ${ }^{[6]}$ described this as a 'collapse'

\section{Study limitations}

Two limitations were evident in this study. The first is that the frame rate of the cameras used was lower than that used in recent studies, and a higher frame rate would have been ideal. The second limitation was that the data of 14 bowlers could not be included in 
the analysis as a result of missing markers encountered during the 3D kinematic analysis, mainly due to the high-speed bowling action.

\section{Conclusion}

Body actions that result in higher BR speeds (enhanced performance) have raised concerns among health professionals, since the increased strain on the body that results in greater performance may also increase injury likelihood. The focus on injury prevention in pace bowlers is vital, but it cannot be examined in isolation. In the investigation of variables related to injury, the need for optimal bowling performance should also be taken into account. In addition, knee angle should be interpreted as part of the kinetic chain and kinematics at the ankle, hip and lower back should be taken into account.

Injury- and bowling performance-related factors associated with the knee classification categories as developed for and used in this study should be further investigated among bowlers. Further research needs to be conducted into non-technique-related strategies to attain higher BR speeds among bowlers who remain injury free during a cricket season. The strategies used to attain higher BR speeds may have contributed to the prevention of injuries.

Acknowledgements. We would like to acknowledge each participant for his time, effort and enthusiasm. Funding for this project was received from the National Research Foundation, the Carnegie Foundation of New York and the local Society of Physiotherapy. These funding organisations played no role in the collection, analysis or interpretation of data and had no right to approve or disprove of the final manuscript.

\section{References}

1. Roca M, Elliott B, Alderson J, Foster D. The relationship between shoulder alignment and elbow joint angle in cricket fast-medium bowlers. J Sports Sci 2006;24(11):11271135. [http://dx.doi.org/10.1080/02640410500497618]

2. Ferdinands RE, Kersting U, Marshall RN. Three-dimensional lumbar segment kinetics of fast bowling in cricket. J Biomech 2009;42(11):1616-1621. [http://dx.doi. org/10.1016/j.jbiomech.2009.04.035]
3. Crewe H, Campbell A, Elliott B, Alderson J. Lumbo-pelvic loading during fast bowling in adolescent cricketers: The influence of bowling speed and technique. J Sports Sci 2013;31(10):1082-1090. [http://dx.doi.org/10.1080/02640414.2012.762601]

4. Portus M, Mason BR, Elliott BC, Pfitzner MC, Done RP. Technique factors related to ball release speed and trunk injuries in high performance cricket fast bowlers. Sports Biomech 2004;3(2):263-284. [http://dx.doi. org/10.1080/14763140408522845]

5. Wormgoor S, Harden L, McKinon W. Anthropometric, biomechanical, and isokinetic strength predictors of ball release speed in high-performance cricket fast bowlers. J Sports Sci 2010;28(9):957-965. [http://dx.doi. org/10.1080/02640411003774537]

6. Bartlett RM, Stockill NP, Elliott BC, Burnett AF. The biomechanics of fast bowling in men's cricket: A review. J Sports Sci 1996;14(5):403-424. [http://dx.doi. org/10.1080/02640419608727727]

7. Portus MR, Sinclair PJ, Burke ST, Moore DJ, Farhart PJ. Cricket fast bowling performance and technique and the influence of selected physical factors during an 8-over spell. J Sports Sci 2000;18(12):999-1011. [http://dx.doi. org/10.1080/026404100446801]

8. Zhang Y, Unka J, Liu G. Contributions of joint rotations to ball release speed during cricket bowling: A three-dimensional kinematic analysis. J Sports Sci 2011;29(12):1293-1300. [http://dx.doi.org/10.1080/02640414.2011.591417]

9. Elliott BC, Hardcastle PH, Burnett AE, Foster DH. The influence of fast bowling and physical factors on radiologic features in high performance young fast bowlers. Sports Med Train Rehabil 1992;3(2):113-130.

10. Orchard J, James T, Alcott E, Carter S, Farhart P. Injuries in Australian cricket at first class level 1995/1996 to 2000/2001. Br J Sports Med 2002;36(4):270-274;discussion 5 .

11. Stretch R, Raffan R. Injury patterns of South African international cricket players over a two-season period. S Afr J Sports Med 2011;23(2):45-49.

12. Frost WL, Chalmers DJ. Injury in elite New Zealand cricketers 2002-2008: Descriptive epidemiology. Br J Sports Med 2014;48(12):1002-1007. [http://dx.doi.org/10.1136/ bjsports-2012-091337]

13. Orchard J, Newman D, Stretch R, Frost W, Mansingh A, Leipus A. Methods for injury surveillance in international cricket. J Sci Med Sport 2005;8(1):1-14.

14. Olivier B, Stewart AV, Olorunju SA, McKinon W. Static and dynamic balance ability, lumbo-pelvic movement control and injury incidence in cricket pace bowlers. J Sci Med Sport 2015;18(1):19-25.

15. Portney LG, Watkins MP. Foundations of Clinical Research: Applications to Practice. London: Pearson Prentice Hall, 2009.

16. Putnam CA. Sequential motions of body segments in striking and throwing skills: Descriptions and explanations. J Biomech 1993;26 Suppl 1:125-135.

17. Olivier B, Stewart AV, McKinon W. Injury and lumbar reposition sense in cricket pace bowlers in neutral and pace bowling specific body positions. Spine J 2014;14:14471453. [http://dx.doi.org/10.1016/j.spinee.2013.08.036] 